\title{
Why the emperor penguin reigns where elephants shiver
}

\author{
Ana Cristina Nogueira Freitas and Thomas Voets \\ Laboratory of Ion Channel Research (LICR), VIB-KU Leuven Centre for Brain \& Disease Research, \\ Leuven, Belgium \\ and \\ Department of Cellular and Molecular Medicine, KU Leuven, Leuven, Belgium
}

\begin{abstract}
Accurate sensing of the environmental temperature is crucial for the survival and wellbeing of organisms. In vertebrates, the cold- and menthol-activated ion channel TRPM8 acts as the prime molecular sensor of cool temperatures. By comparing TRPM8 in vertebrates with different habitat temperatures, from elephants to penguins, Yang et al. identify key residues within the pore domain that determine the channel's cold sensitivity. Strikingly, mice engineered to express penguin TRPM8 show a remarkable tolerance to cold.
\end{abstract}

Environmental temperature is a critical factor that has a great impact on the integrity and metabolism of all living beings. The invisible hand of natural selection caused plants and animals to develop specific ecological and physiological features that enable them to cope with the temperature oscillations in their specific environment. For instance, the thermal challenges encountered by a lion inhabiting the Africa savannas differ substantially from those encountered by a polar bear in the arctic.

The temperature-sensitive peripheral neurons of the somatosensory system constantly convey information regarding the environmental temperature to the central nervous system. Undoubtedly, the discovery and characterization of temperaturesensitive ion channels of the transient receptor potential (TRP) superfamily - so called thermoTRPs - represented a great leap forward in our understanding of how the thermal stimuli are perceived [1]. In mammals, thermoTRPs are expressed in the free endings of temperature-sensitive neurons in the skin, where their activity is steeply dependent on the local temperature. As such, thermoTRPs act as the molecular thermometers that translate changes in temperature into nerve activity.

Given the importance of thermosensitivity, it is not surprising that thermoTRPs channels can be found all phyla of the animal kingdom, from the tiny fruit fly to the largest mammal on land, the elephant [2, 3]. However, considering the broad range of thermal environments encountered on earth, from below $-50^{\circ} \mathrm{C}$ on the poles to above $+50^{\circ} \mathrm{C}$ in the Sahara, specific evolutionary adaptations in the thermal tuning of thermoTRPs can be expected.

Among the thermoTRPs, it is not a matter of debate that TRPM8 plays a central role in cold sensing. Notably, TRPM8 is not only activated upon cooling, but also by a variety of natural and synthetic ligands that evoke a cooling sensation, of which menthol is the best known. Recently, new insights into the molecular mechanism of TRPM8 activation were obtained from high-resolution structures of the channel in the absence and presence of different activating ligands [4]. Yet, the structural 
rearrangements that underlie activation of TRPM8 during cooling still need to be fully clarified.

In their recent study, Yang et al. used a fascinating approach to elucidate the molecular framework of TRPM8 activation upon cooling. They started by functionally comparing the temperature sensitivity of TRPM8 orthologs found in vertebrates species inhabiting a broad spectrum of habitat temperatures. They observed that the cold responses of the different orthologs channels positively correlates with the habitat temperature of the respective animals. Among all tested orthologs (including human), the one that presented the most robust response to cooling originates from the African elephant Loxodonta africana (TRPM8_LA), an inhabitant of the hot Sahel desert. On the other side of the spectrum, the emperor penguin, Aptenodytes forsteri, which is only found in the ice-cold Antarctic region, presented the least cold-sensitive TRPM8 (TRPM8_AF) ortholog. The sensitivity of human TRPM8 is somewhere in the middle (Figure 1A). It thus seems that coping with extreme cold is facilitated by a thermosensor that is tuned towards colder temperatures (Figure $1 \mathrm{~A}$ ).

Yang et al. used these functional differences to elucidate elements in the channel that underlie cold sensitivity. Importantly, by swapping non-conserved residues of the TRPM8 pore domain (PD) between TRPM8_LA and TRPM8_AF, they identified a single amino acid residue at position 919 that has a great impact on the thermal sensitivity of the channel. Introducing the penguin residue at this position into TRPM8_LA (V919Y) strongly reduced the response to cold, whereas the reverse mutation in TRPM8_AF (Y919V) induced a robust increase of cold sensitivity (Figure 1B). In contrast, interchanging non-conserved residues outside the PD had almost no impact on cold sensitivity.

It has been suggested that temperature sensitivity of ion channels may be profoundly influenced by the solvation of the side chains of residues that become exposed during gating. For instance, increasing the hydrophobicity of such key residues could lead to an increase of the cold response [5]. Following this concept, Yang et al. delineate three key residues that are supposedly clustered in the PD according to computational modeling, which may transit from a buried state to a waterexposed state during channel gating. Point mutations of these three residues (at positions 925, 943 and 947) along with the previously discussed position 919, showed that an increase in the hydrophobicity of these residues causes enhanced cold sensitivity. Interestingly, the hydrophobicity content of the residues within the TRPM8 PD appears perfectly tuned with thermal adaptation, since it positively correlates with habitat temperature and maximum cold response of the different species. Note that, although this study concludes that key determinants of cold sensitivity are found in the PD of TRPM8, other regions of the channel may also influence thermosensitivity, such as the $\mathrm{C}$ terminus or the voltage sensor domain $[6,7]$.

Finally, Yang et al. beautifully illustrated the critical contribution of TRPM8 to thermal adaptation by generation of mice in which the TRPM8 gene was replaced by the gene encoding TRPM8_AF. These mice-with-a-penguin-cold-sensor preferred cooler temperatures and were more tolerant to cold when compared to wild-type mice. Therefore, the gene encoding TRPM8 defines temperature preference (Figure 1C), 
and was likely of great influence on the thermal evolution of vertebrates inhabiting the wide range of habitat-temperature all over the world [8-10].

\section{References}

[1] J. Vriens, B. Nilius, T. Voets, Peripheral thermosensation in mammals, Nat Rev Neurosci, 15 (2014) 573-589.

[2] S. Saito, M. Tominaga, Functional diversity and evolutionary dynamics of thermoTRP channels, Cell Calcium, 57 (2015) 214-221.

[3] S. Yang, X. Lu, Y. Wang, L. Xu, X. Chen, F. Yang, R. Lai, A paradigm of thermal adaptation in penguins and elephants by tuning cold activation in TRPM8, Proc Natl Acad Sci U S A, 117 (2020) 8633-8638.

[4] Y. Yin, S.C. Le, A.L. Hsu, M.J. Borgnia, H. Yang, S.Y. Lee, Structural basis of cooling agent and lipid sensing by the cold-activated TRPM8 channel, Science, 363 (2019).

[5] S. Chowdhury, B.W. Jarecki, B. Chanda, A molecular framework for temperature-dependent gating of ion channels, Cell, 158 (2014) 1148-1158.

[6] S. Brauchi, G. Orta, M. Salazar, E. Rosenmann, R. Latorre, A hot-sensing cold receptor: C-terminal domain determines thermosensation in transient receptor potential channels, J Neurosci, 26 (2006) 4835-4840.

[7] T. Voets, G. Owsianik, A. Janssens, K. Talavera, B. Nilius, TRPM8 voltage sensor mutants reveal a mechanism for integrating thermal and chemical stimuli, Nat Chem Biol, 3 (2007) 174-182.

[8] V. Matos-Cruz, E.R. Schneider, M. Mastrotto, D.K. Merriman, S.N. Bagriantsev, E.O. Gracheva, Molecular Prerequisites for Diminished Cold Sensitivity in Ground Squirrels and Hamsters, Cell Rep, 21 (2017) 3329-3337.

[9] B.R. Myers, Y.M. Sigal, D. Julius, Evolution of thermal response properties in a cold-activated TRP channel, PLoS One, 4 (2009) e5741.

[10] M. Pertusa, B. Rivera, A. Gonzalez, G. Ugarte, R. Madrid, Critical role of the pore domain in the cold response of TRPM8 channels identified by ortholog functional comparison, J Biol Chem, 293 (2018) 12454-12471. 
A)

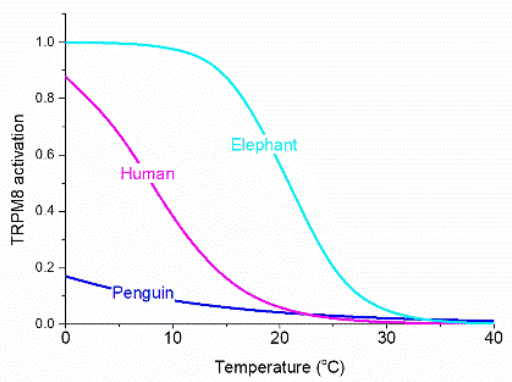

B)

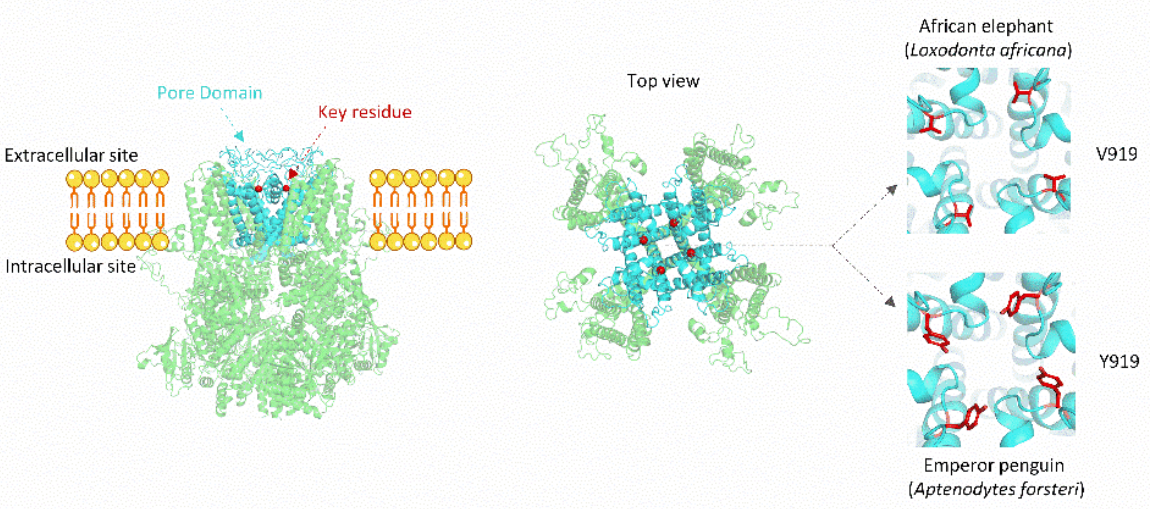

C)

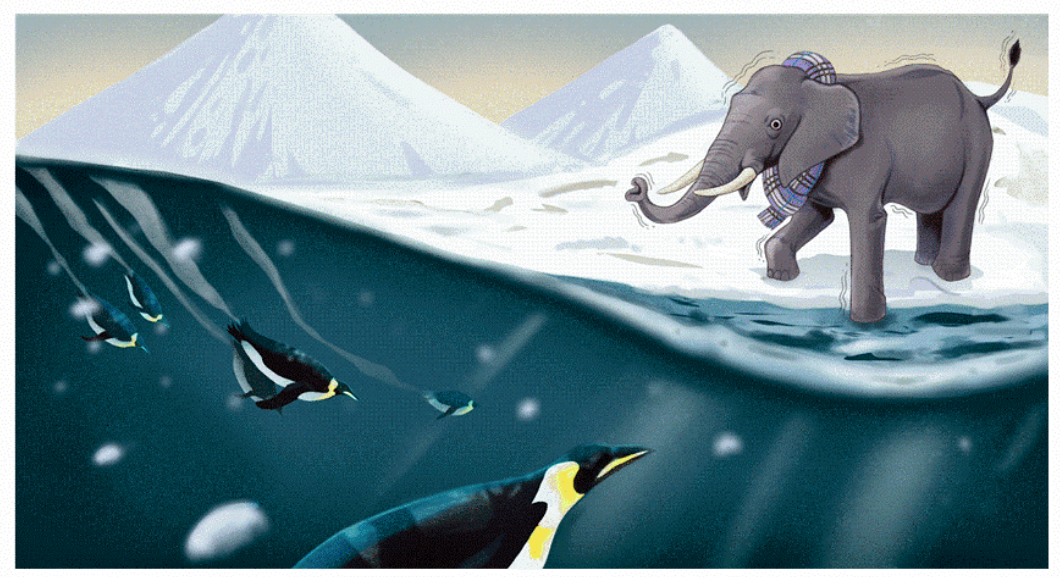

Figure 1

A, Temperature dependence of the activation of TRPM8, a major cold sensor in the somatosensory system, for the orthologs from the African elephant Loxodonta Africana, human, and the emperor penguin, Aptenodytes forsteri. Activation curves were calculated based on thermodynamic parameters described in refs. 3 and 7 . B, A critical amino acid residue in the pore domain of TRPM8 underlies the difference in thermal sensitivity between elephant and penguin. $\mathbf{C}$, The evolutionary differences in thermal sensitivity likely contributed to how animals cope with cold environments (Illustration by Fred Freitas). 